\title{
On conjugate points and the Leitmann equivalent problem approach $^{\widehat{\hbar}}$
}

\author{
F.O.O. Wagener ${ }^{\mathrm{a}, 1}$ \\ ${ }^{a}$ CeNDEF, Department of Quantitative Economcis, Universiteit van Amsterdam, Roetersstraat 11, \\ 1018 WB, Amsterdam, The Netherlands
}

\begin{abstract}
This article extends the Leitmann equivalence method to a class of problems featuring conjugate points. The class is characterised by the requirement that the set of indifference points of a given problem forms a finite stratification.
\end{abstract}

Key words: Calculus of variations, equivalent problems, conjugate points, rectifying coordinates, sufficient conditions

\section{Dedicated to George Leitmann}

\section{Introduction}

In this article, the Leitmann equivalence method $[1,2,3]$ that gives absolute extrema of calculus of variations problems is extended to a class of problems that feature conjugate points.

Recall that the Leitmann equivalence method consists in considering a classical field of extremals as a coordinate transformation: the extremals of the transformed problem are then constants. Optimality of the transformed extremals is obtained by using Carathéodory's equivalent problem approach [4]. This gives the sufficiency theory of the classical Calculus of Variations a particularly simple and elegant form.

However, the above summary of the equivalence method also indicates one of its main limitations: the method breaks down when extremals intersect, that is, when the field of extremals fails to define an invertible coordinate transformation. It may fail globally, when a point in the extended state space is reached by several extremals, or locally, when the differential of the transformation at a point fails to have full rank. The latter points are classically called conjugate.

\footnotetext{
Running title: On conjugate points

Email address: f.o.०.wagener@uva.nl (F.O.O. Wagener)

${ }^{1}$ The author would like to thank George and Nancy Leitmann for helpful remarks, illuminating discussions and warm hospitality during the preparation of this paper. This work has been supported by NWO under a MaGW-VIDI grant.
} 
The present article is a step in the direction of removing this limitation. First, only the extremals of the field are considered. The obvious but important thing to note here is that an extremal should be restricted to its maximal domain of optimality, which is defined as the maximal interval of definition such that no other extremal in the field having the same endpoint gives a lower value to the objective functional. If the domain of optimality does not coincide with the integration domain of the objective functional, several situations may arise: either the extremal cannot be extended to a larger domain of definition or it fails to be optimal on a larger domain. In the latter situation, the endpoint is either a conjugate point, an indifference point, meaning that it is reached by several extremals all giving the objective functional the value, or it may be an infimal point: in this case, the point is reached by an infinity of extremals, but the set of associated values of the objective functional has no minimal value.

Attention is restricted to the situation that there are no infimal points, and that the set of indifference points forms a finite stratification; this means that it is the union of finitely many open differential manifolds, possibly of different dimensions, such that each manifold that intersects the closure of another manifold is actually contained in this closure. Having specified in this way the structure of the set of extremals restricted to their domains of optimality, the second step is to show that an element of this set actually minimises the objective functional also on the much larger set of all admissible trajectories. In the proof of this second step, it is sufficient to consider the generic situation of a smooth non-extremal trajectory attaining a lower value of the objective functional than all extremals of the field, and intersecting only finitely many indifference manifolds finitely often. The main technical point then is to show how these intersections can be removed without changing the value of the objective functional too much, ending up with a trajectory that has no intersections with any indifference manifold, but still realises a lower value of the objective functional than any extremal. Leitmann rectification now immediately shows the impossibility of this situation. The theorem is illustrated by its application to a relatively simple problem that features indifference points.

Leitmann's rectifying coordinates are closely related to Kneser's normal coordinates of a field. Kneser [5], §22, considered parametric problems having a positive integrand. He also used a field of extremals as a coordinate transformation; however, as the second normal variable he took the accumulated value of the objective functional along the extremal. This ensures that the integrand of the objective functional is constant along extremals and it eliminates the need to consider an equivalent problem. In $\$ 22 . I V$ Kneser demonstrates that in normal coordinates, the resulting variational problem can be solved by inspection. The restriction of Kneser to problems with positive integrands was forced by the need to have the integrand transform to a simple form, as the method of equivalent problems was not known at the time. The central idea of the present article, to show that for the problem of finding a global minimum attention can be restricted to the extremal trajectories, has its roots in the so-called Calculus of Variations in the Large, where, however, it is applied to several relatively short parts of the non-extremal trajectories [see 4, §385].

There is a rapidly growing literature on the subject of Leitmann rectification, showing that the method is general and in principle applicable to all kinds of problems connected to the Calculus of Variations; see [2, 3, 6, 7, 8, 9, 10, 11, 12]. 


\section{The problem}

\subsection{Preliminary definitions.}

In the following, any $\mathscr{C}^{k}$ function defined on a closed set $G$ is always assumed to be the restriction of a $\mathscr{C}^{k}$ function defined on an open neighbourhood of $G$.

Let points $a, b \in \mathbb{R}$ be given, $a<b$, as well as points $\alpha, \beta \in \mathbb{R}^{n}$. The set $\mathcal{X}=$ $[a, b] \times \mathbb{R}^{n}$ is called the extended state space. Let $L: \mathcal{X} \times \mathbb{R}^{n} \rightarrow \mathbb{R}$ be a $\mathscr{C}^{2}$ function on the extended tangent space $\mathcal{T}=\mathcal{X} \times \mathbb{R}^{n}$. Writing $L=L(t, x, v)$ with $(t, x) \in \mathcal{X}$ and $v \in \mathbb{R}^{n}$, it is assumed that

$$
L_{v v}(t, x, v)>0 \quad \text { for all }(t, x) \in \mathcal{X} .
$$

Finally, for $a<T \leq b$, let $\mathscr{A}_{T}$ be the space of absolutely continuous functions $x$ : $[a, T] \rightarrow \mathbb{R}^{n}$ that are such that $x(a)=\alpha$; an element of $\mathscr{A}_{T}$ will be called a trajectory (starting in $\alpha$ ) in the following. Let moreover $\mathscr{B}_{T, X}$ be the subset of $\mathscr{A}_{T}$ of trajectories $x$ that satisfy $x(T)=X$.

The space $\mathscr{A}_{T}$ will be equipped with the topology induced by the metric

$$
d_{T}\left(x_{1}, x_{2}\right)=\int_{a}^{T}\left|\dot{x}_{1}(t)-\dot{x}_{2}(t)\right| \mathrm{d} t .
$$

Recall that the set of $\mathscr{C}^{\infty}$ trajectories is dense in $\mathscr{A}_{T}$ with respect to this metric.

\subsection{The minimisation problem.}

Introduce for $a<T \leq b$ the functional $J_{T}: \mathscr{A}_{T} \rightarrow \mathbb{R}$ that is defined by

$$
J_{T}(x)=\int_{a}^{T} L(t, x, \dot{x}) \mathrm{d} t .
$$

In this article, I consider the standard problem to find a minimiser of $J_{b}$ on $\mathscr{B}_{b, \beta}$, that is, an element $x_{0} \in \mathscr{A}_{b}$ such that $x_{0}(b)=\beta$ and such that $J_{b}\left(x_{0}\right) \leq J_{b}(x)$ for all $x \in \mathscr{B}_{b, \beta}$.

Recall from the Calculus of Variations that if $x \in \mathscr{B}_{b, \beta}$ minimises $J_{b}$, then it is necessarily a solution to the Euler-Lagrange equation

$$
L_{x}(t, x, \dot{x})-\frac{\mathrm{d}}{\mathrm{d} t} L_{v}(t, x, \dot{x})=0,
$$

satisfying the boundary conditions

$$
x(a)=\alpha, \quad x(b)=\beta .
$$

In general, solutions to (1) are called extremals. The regularity assumption $L_{v v}>0$ implies that every extremal is at least $\mathscr{C}^{2}$. Introduce the subspaces $\mathscr{E}_{T} \subset \mathscr{A}_{T}$ and $\mathscr{E}_{T, X} \subset$ $\mathscr{B}_{T, X}$ of trajectories in $\mathscr{A}_{T}$ and $\mathscr{B}_{T, X}$ respectively that are extremals. 


\section{Leitmann rectification.}

Leitmann has developed a method which replaces the problem to minimise $J_{T}$ by the problem to minimise an equivalent functional $\tilde{J}_{T}$, whose minimisers can be determined "by inspection". The method consists, if it works, in finding a field, that is, an $n$-parameter family of extremals satisfying a certain integrability condition, such that each point in the extended state space is covered by exactly one extremal. Using this family as a coordinate transformation, the extremals transform to straight lines, the Hamilton-Jacobi partial differential equation simplifies to a family of simple ordinary differential equation solvable by quadratures, and the transformed problem can be solved by an easy application of Carathéodory's "royal road" approach.

That the method does not always work is a consequence of the well-known fact that (the graphs of) extremals may intersect; this precludes the existence of globally defined rectifying coordinates. However, if two extremals $x_{1}, x_{2}$ intersect at a point $(t, x)$, then that extremal which gives the larger value of $J_{t}$ should obviously be discarded. If both extremals yield the same value, and no other extremal yields a smaller value, the point $(t, x)$ is called an indifference point. The natural domain of definition of the Leitmann equivalence transformation is therefore the open set that has the union of the set of indifference points and the terminal line $t=b$ as its boundary.

\subsection{Domain of injectivity.}

Let us formulate these ideas more precisely. Denote by

$$
t \mapsto \xi(t, y)
$$

the extremal that satisfies the boundary conditions $\xi(a, y)=\alpha, \xi_{t}(a, y)=y$. The maximal domain of definition of $\xi(\cdot, y)$ is denoted by $\left[a, \omega_{y}\right)$. Note that the family $\xi(t, y)$ forms a central field of extremals [cf. 12].

The value of the integral along $\xi(\cdot, y)$ is given as

$$
v(t, y)=J_{t}(\xi(\cdot, y))=\int_{a}^{t} L\left(s, \xi(s, y), \xi_{s}(s, y)\right) \mathrm{d} s .
$$

If $(t, x)=\xi(t, y)$, and if $\xi_{y}(t, y) \neq 0$, then there is an open neighbourhood $\tilde{U}$ of $(t, y)$ that is mapped diffeomorphically to a neighbourhood $U$ of $(t, x)$ by $(t, y) \mapsto(t, \xi(t, y))$. Define $W: U \rightarrow \mathbb{R}$ by

$$
W(t, \xi(t, y))=v(t, y),
$$

that is, $W(t, x)=v(t, y)$ whenever $x=\xi(t, y)$.

Lemma 1. If $\xi_{y}$ is invertible at $(t, y)$, then

$$
W_{x}(t, \xi(t, y))=L_{v}\left(t, \xi(t, y), \xi_{t}(t, y)\right) .
$$

Proof. Differentiate (2) with respect to $y$ to obtain

$$
W_{x}(t, \xi(t, y)) \xi_{y}=\int_{a}^{t}\left(L_{x} \xi_{y}+L_{\nu} \xi_{s y}\right) \mathrm{d} s .
$$


Here and in the following, arguments $(t, y)$ of $\xi$ and $\left(t, \xi(t, y), \xi_{t}(t, y)\right)$ of $L$ may be omitted without explicit mention. Integrating partially, and using that $\xi(., y)$ satisfies the Euler-Lagrange equation as well as the fact that $\xi(a, y)=\alpha$ for all $y$ and hence that $\xi(y, a, y)=0$, yields

$$
\begin{aligned}
W_{x}(t, \xi(t, y)) \xi_{y} & =\int_{a}^{t}\left(L_{x}-\frac{\mathrm{d}}{\mathrm{d} t} L_{v}\right) \xi_{y} \mathrm{~d} s+\left.L_{v} \xi_{y}\right|_{a} ^{t} \\
& =L_{v}\left(t, \xi(t, y), \xi_{t}(t, y)\right) \xi_{y} .
\end{aligned}
$$

Multiplication with the inverse of $\xi_{y}$ yields the result.

Let $\mathcal{Y}_{(t, x)}$ be the set of parameters $y$ that are such that $\xi(t, y)=x$. Introduce also the function $V(t, x)$ by setting

$$
V(t, x)=\inf \left\{v(t, y) \mid y \in \mathcal{Y}_{(t, x)}\right\} .
$$

Define a set $\tilde{\mathcal{D}} \subset[a, b] \times \mathbb{R}^{n}$ as follows. If the infimum in the definition of $V(t, x)$ is actually a minimum that is realised by a unique element $y \in \mathcal{Y}_{(t, x)}$, then $(t, y) \in \tilde{\mathcal{D}}$.

It follows from the principle of optimality that if $\left(t_{0}, y\right) \notin \tilde{\mathcal{D}}$, then $(t, y) \notin \tilde{\mathcal{D}}$ for all $t>t_{0}$. Introduce

$$
\tau_{y}=\sup \left\{t \in\left[a, \omega_{y}\right) \mid(t, y) \in \tilde{D}\right\} .
$$

Note that if $t=\tau_{y}$, then necessarily either $t=\omega_{y}$, or there exists $\bar{y} \in \mathbb{R}^{n}$ such that $\xi(t, y)=\xi(t, \bar{y})$ and $v(t, y)=v(t, \bar{y})$, or there is an infinite sequence $\left\{y_{n}\right\}$ such that

$$
\xi\left(t, y_{1}\right)=\xi\left(t, y_{2}\right)=\cdots
$$

and

$$
v\left(t, y_{1}\right)>v\left(t, y_{2}\right)>\cdots
$$

\subsection{The rectification map.}

Define the map

$$
\Xi: \tilde{\mathcal{D}} \rightarrow \mathbb{R} \times \mathbb{R}^{n}, \quad(t, y) \mapsto \Xi(t, y)=(t, \xi(t, y)) .
$$

Define the domain of unicity by setting $\mathcal{D}=\Xi(\tilde{\mathcal{D}})$. A point $(t, x)$ is in $\mathcal{D}$ if and only if there is a unique extremal joining $(a, \alpha)$ and $(t, x)$ minimising $J_{t}$ over the set of all extremals $\xi(\cdot, y)$ with $y \in \mathcal{Y}_{(t, x)}$; that is, there is a unique element $y \in \mathcal{Y}_{(t, x)}$ that minimises

$$
j_{t}(y)=J_{t}(\xi(\cdot, y)) \text {. }
$$

For these points, a map $\xi^{-1}(t, \cdot)$ is defined by requiring that

$$
\xi\left(t, \xi^{-1}(t, x)\right)=x
$$

A point $(t, x)$ is in the relative complement $X \backslash \mathcal{D}$ of $\mathcal{D}$ if one of two possibilities hold. The first possibility is that there are at least two elements $y_{1}, y_{2} \in \mathcal{y}_{(t, x)}$ that yield the minimum value of $j_{t}$ : such a point is called an indifference point. The second 
possibility is that the set of values $j_{t}\left(\mathcal{Y}_{(t, x)}\right)$ has no minimal element; that is, there are infinitely many extremals joining $(a, \alpha)$ to $(t, x)$, and the set of values of $J_{t}$ over these extremals has no minimal element. In this case, the point $(t, x)$ will be called infimal.

Recall also the definition of conjugate states: a state $\left(t_{0}, x_{0}\right)$ is conjugate to $(a, \alpha)$ along the extremal $\xi\left(., y_{0}\right)$, if there are sequences $y_{k}^{(1)}, y_{k}^{(2)} \rightarrow y_{0}$ with $y_{k}^{(1)} \neq y_{k}^{(2)}$ for any $n$, and a sequence $\left(t_{k}, x_{k}\right)$, such that

$$
\left(t_{k}, x_{k}\right)=\xi\left(t_{k}, y_{k}^{(1)}\right)=\xi\left(t_{k}, y_{k}^{(2)}\right)
$$

and such that

$$
\left(t_{k}, x_{k}\right) \rightarrow\left(t_{0}, x_{0}\right)
$$

as $n \rightarrow \infty$. It follows from this definition that $\xi_{y}$ cannot be invertible at $\left(t_{0}, y_{0}\right)$ :

$$
\operatorname{det} \xi_{y}\left(t_{0}, y_{0}\right)=0
$$

The set of indifference points is denoted by $\mathcal{I}$; the set of conjugate points by $C$. Necessarily $\partial \mathcal{I} \subset C$.

The following theorem is already contained in Weierstrass' work on strong minima [13]; Leitmann's rectification method furnishes a very elegant proof (see [1, 2, 12, 14]).

Theorem 1. Let $\tilde{x} \in \mathscr{B}_{b, \beta}$ be such that $(t, \tilde{x}(t)) \in \mathcal{D}$ for all $a<t \leq b$, and let $x \in \mathscr{E}_{b, \beta}$ be an extremal, necessarily unique, that satisfies $(t, x(t)) \in \mathcal{D}$ for all $a<t \leq b$. Moreover, assume that $x$ and $\tilde{x}$ are not identical. Then

$$
J(\tilde{x})>J(x) .
$$

\section{Main theorem}

In this section, the main theorem of the paper is stated and proved.

Recall the definition of a stratification: a disjoint collection $\mathscr{S}=\left\{\mathcal{S}_{i}: i=1, \cdots\right\}$ of embedded submanifolds $\mathcal{S}_{i}$ is a stratification, and its union $\mathcal{S}=\cup_{i} \mathcal{S}_{i}$ is a stratified set, if $\mathcal{S}_{i} \cap \overline{\mathcal{S}_{j}} \neq \emptyset$ implies $\mathcal{S}_{i} \subset \overline{\mathcal{S}_{j}}$. The stratification is finite if the collection $\mathscr{S}$ is finite.

Assumption 1. In the following, it is assumed that there are no infimal points, that the set of indifference points $\mathcal{I}$ is a finitely stratified set, with stratification $\left\{\mathcal{I}_{1}, \cdots, \mathcal{I}_{\ell}\right\}$, and that the set $\overline{\mathcal{I}} \cap C$ of those conjugate points that are contained in the closure of the set of indifference points is a finite stratification of manifolds that have at most dimension $n-1$.

Introduce

$$
\mathcal{Y}_{(t, x)}^{\min }=\left\{y \in \mathcal{Y}_{(t, x)} \mid j_{t}(y) \leq j_{t}(z) \text { for all } z \in \mathcal{Y}_{(t, x)}\right\}
$$

It is a consequence of the assumption that $\mathcal{Y}_{(t, x)}^{\min } \neq \emptyset$ for any $(t, x) \in \mathcal{X}$. Consequently, the function $V$ is defined everywhere on $\mathcal{X}$. 
Theorem 2. Let assumption 1 hold. If $x(t)=\xi(t, y)$ is an extremal for which $y \in \mathcal{Y}_{(b, \beta)}^{\min }$, then x minimises $J_{b}$ over $\mathscr{B}_{b, \beta}$.

Proof. Arguing by contradiction, assume that there is a trajectory $x_{-2} \in \mathscr{B}_{b, \beta} \backslash \mathscr{E}_{b, \beta}$ such that

$$
J_{b}\left(x_{-2}\right)<m=\min _{x \in \mathscr{E}_{b, \beta}} J_{b}(x) .
$$

Noting that $J_{b}: \mathscr{B}_{b, \beta} \rightarrow \mathbb{R}$ is continuous with respect to the topology introduced in subsection 2.1, and making use of the fact that the $\mathscr{C}^{\infty}$ elements of $\mathscr{B}_{b, \beta}$ are dense in $\mathscr{B}_{b, \beta}$ with respect to the same topology, it follows that there is a $\mathscr{C}^{\infty}$ trajectory $x_{-1}$ for which also

$$
J_{b}\left(x_{-1}\right)<m .
$$

From the weak transversality theorem it follows that there is a $\mathscr{C}^{\infty}$ trajectory $x_{0}$, such that the curve $t \mapsto\left(t, x_{0}(t)\right)$ intersects every manifold $\mathcal{I}_{i}$ of the stratification of $\mathcal{I}$ transversally, as well as the manifolds of the stratification of $\overline{\mathcal{I}} \cap \mathcal{C}$, and such that

$$
J_{b}\left(x_{0}\right)<m \text {. }
$$

Since the maximal dimension of manifolds in the stratification of $\overline{\mathcal{I}} \cap C$ is $n-1$, it follows from transversality that $x_{2}$ does only intersect the $n$-dimensional manifolds in the stratification $\mathcal{I}$, and it does not intersect $\overline{\mathcal{I}} \cap C$ at all.

In the following it will be shown that the existence of $x_{0}$ implies that there is another $\mathscr{C}^{\infty}$ trajectory $x_{N}$ in $\mathscr{B}_{b, \beta}$ that satisfies $J_{b}\left(x_{N}\right)<m$, and that does not intersect $\mathcal{I}$ at all. But this will lead to a contradiction.

As $x_{0}$ intersects each $\mathcal{I}_{i}$ transversally, these intersections are isolated, and hence the number of such intersections is finite; let this number be $N_{i}$. Consequently, the total number $N=\sum N_{i}$ of intersections of $x_{0}$ with $\mathcal{I}$ is finite as well. We shall perform an induction on the number of intersections, showing that for a given trajectory with a finite number of transversal intersections with $\mathcal{I}$, it is always possible to find a trajectory with a smaller number of intersections, which also yields a value of the functional smaller than $m$. The induction ends with a trajectory that is entirely contained in $\mathcal{D}$, yielding a value of the objective functional smaller than $m$; but by theorem 1 , the trajectory is shown to yield a value of the functional at least as large as $m$, resulting in a contradiction.

Assume therefore that there is a $\mathscr{C}^{\infty}$ trajectory $x_{k}$, such that $x_{k}$ is transversal to $\mathcal{I}$ and such that it does not intersect $\bar{I} \cap C$ at all, having $N-k$ points of intersection with $\mathcal{I}$ at the intersection times $t_{1}, t_{2}, \cdots, t_{N-k}$, and such that

$$
J\left(x_{k}\right)<m .
$$

Let $(\bar{t}, \bar{x})=\left(t_{1}, x_{k}\left(t_{1}\right)\right) \in \mathcal{I}$ be the first intersection of $x_{k}$ with $\mathcal{I}$. Since $(\bar{t}, \bar{x})$ is an indifference point, there are $y_{1}, y_{2} \in \mathcal{Y}_{(\bar{t}, \bar{x})}, y_{1} \neq y_{2}$, such that

$$
v\left(\bar{t}, y_{1}\right)=v\left(\bar{t}, y_{2}\right)=V(\bar{t}, \bar{x}) .
$$

Since $(\bar{t}, \bar{x})$ is in $\mathcal{I}$ but not in $\overline{\mathcal{I}} \cap \mathcal{C}$, it follows that

$$
\operatorname{det} \xi_{y}\left(t, y_{1}\right) \neq 0 \neq \operatorname{det} \xi_{y}\left(t, y_{2}\right) \text {. }
$$


Then there are open neighbourhoods $U_{1}$ and $U_{2}$ of $y_{1}$ and $y_{2}$ respectively, and an $\varepsilon>0$, such that $\Xi(t, y)=(t, \xi(t, y))$ maps $U_{1}$ and $U_{2}$ both diffeomorphically onto the open set

$$
B=(\bar{t}-\varepsilon, \bar{t}+\varepsilon) \times B_{\varepsilon}(\bar{x}) \subset(a, b) \times \mathbb{R}^{n} .
$$

Define on $B$ the functions $V_{1}, V_{2}: B \rightarrow \mathbb{R}$ by

$$
V_{i}(t, \xi(t, y))=v(t, y), \quad \text { for all }(t, y) \in U_{i}, \quad i=1,2 .
$$

Lemma 1 implies that

$$
\frac{\partial V_{i}}{\partial x}(\bar{t}, \bar{x})=L_{v}\left(t, \xi\left(t, y_{i}\right), \xi_{t}\left(t, y_{i}\right)\right) .
$$

Introduce

$$
\Delta(t, x)=V_{2}(t, x)-V_{1}(t, x)
$$

Then

$$
B \cap \mathcal{I}=\{(t, x) \in B \mid \Delta(t, x)=0\} .
$$

Note that for $(t, x) \in B \cap I$

$$
\Delta_{x}=\frac{\partial V_{2}}{\partial x}-\frac{\partial V_{1}}{\partial x} \neq 0 ;
$$

for if this is not the case, then

$$
\xi\left(\bar{t}, y_{1}\right)=\xi\left(\bar{t}, y_{2}\right)=\bar{x}
$$

as well as

$$
L_{v}\left(t, \bar{x}, \xi_{t}\left(t, y_{1}\right)\right)=L_{v}\left(t, \bar{x}, \xi_{t}\left(t, y_{2}\right)\right) .
$$

Since $L_{v v}$ is invertible everywhere, this equation can be solved for

$$
\xi_{t}\left(t, y_{1}\right)=\xi\left(t, y_{2}\right) ;
$$

But two solutions of the second-order Euler-Lagrange equation whose values and first derivatives coincide at a given point are identical, implying that $y_{1}=y_{2}$, contrary to our assumption. Hence inequality (3) has to hold.

Let $\delta(t)=\Delta\left(t, x_{k}(t)\right)$, and note that $\delta(\bar{t})=0$. Because of transversality, $\delta^{\prime}(\bar{t}) \neq 0$; in fact, taking $\varepsilon>0$ sufficiently small, it may be assumed that if $\left(t, x_{k}(t)\right) \in B$, then

$$
\delta^{\prime}(t)>0 .
$$

It follows that if $\left(t, \xi\left(t, y_{i}\right)\right) \in B$ and $t<\bar{t}$, then

$$
\Delta\left(t, \xi\left(t, y_{1}\right)\right)<0 \quad \text { and } \quad \Delta\left(t, \xi\left(t, y_{2}\right)\right)>0 .
$$

The point $\left(t, x_{k}(t)\right)$ is contained in $\mathcal{D}$ for $a \leq t<\bar{t}$, and it tends to $\bar{x}$ as $t \uparrow \bar{t}$. Therefore, there is an absolutely continuous trajectory $z_{k}$ such that for all $a \leq t<\bar{t}$

$$
x_{k}(t)=\xi\left(t, z_{k}(t)\right)
$$


and $z_{k}(t) \rightarrow y_{1}$ as $t \uparrow \bar{t}$.

From theorem 1 it follows that

$$
J_{\bar{t}}\left(\xi\left(., y_{1}\right)\right) \leq J_{\bar{t}}\left(x_{k}\right)
$$

Define as follows a trajectory $\hat{x}_{k+1}$ that does not cross $\mathcal{I} \cap B$ :

$$
\hat{x}_{k+1}(t)= \begin{cases}\xi\left(t, y_{2}\right) & \text { if } a \leq t \leq \bar{t}, \\ x_{k}(t) & \text { if } \bar{t}<t \leq b .\end{cases}
$$

For all $t$ such that $\left(t, \hat{x}_{k+1}(t)\right) \in B$, it holds that $\Delta\left(\hat{x}_{k+1}(t)\right) \geq 0$. Moreover, since

$$
\tilde{J}_{\bar{t}}\left(\xi\left(., y_{1}\right)\right)=v\left(\bar{t}, y_{1}\right)=v\left(\bar{t}, y_{2}\right)=\tilde{J}_{\bar{t}}\left(\xi\left(., y_{2}\right)\right),
$$

it follows that

$$
J_{b}\left(\hat{x}_{k+1}\right) \leq J_{b}\left(x_{k}\right)<m .
$$

The next step is to modify $\hat{x}_{k+1}$ to a new trajectory $\check{x}_{k+1}$ that has no point in common with $\mathcal{I} \cap B$. Let $\rho>0$ be such that $\bar{t}+\rho<t_{2}-\rho$ and $\hat{x}_{k+1}(t) \in B$ for all $\bar{t}<t<\bar{t}+\rho$; then

$$
\Delta\left(t, \hat{x}_{k+1}(t)\right) \geq 0
$$

for $\bar{t}<t<\bar{t}+\rho$. Let $\tilde{y}_{2}$ be such that $\left(\bar{t}+\rho, \tilde{y}_{2}\right) \in U_{2}$ and

$$
\xi\left(\bar{t}+\rho, \tilde{y}_{2}\right)=\hat{x}_{k+1}(\bar{t}+\rho) .
$$

Introduce $\check{x}_{k+1}$ as follows:

$$
\check{x}_{k+1}(t)= \begin{cases}\xi\left(t, \tilde{y}_{2}\right) & \text { if } a \leq t \leq \bar{t}+\rho, \\ x_{k}(t) & \text { if } \bar{t}+\rho<t \leq b .\end{cases}
$$

The graph of $\check{x}_{k+1}$ is contained in $\mathcal{D}$ for $a \leq t<t_{2}$, and

$$
J\left(\check{x}_{k+1}\right) \leq J\left(\hat{x}_{k+1}\right)<m .
$$

Again by the weak transversality theorem, there is a $\mathscr{C}^{\infty}$ trajectory $x_{k+1}$, having $N$ $(k+1)$ transversal intersections with $\mathcal{I}$, so close to $\check{x}_{k+1}$ in the metric on $\mathscr{A}_{b}$, such that

$$
J\left(x_{k+1}\right)<m \text {. }
$$

This finishes the induction, leading finally to a $\mathscr{C}^{\infty}$ trajectory $x_{N}$ contained entirely in $\mathcal{D}$, and for which

$$
J\left(x_{N}\right)<m \leq J\left(x_{N}\right) .
$$

The second inequality is implied by theorem 1 . As this is a contradiction the theorem has been proved. 


\section{Example}

As an example, consider the problem to minimise

$$
J_{b}(x)=\int_{0}^{b}\left(\frac{\dot{x}^{2}}{2}-\frac{x^{2}}{2}+\frac{x^{4}}{4}\right) \mathrm{d} t,
$$

subject to the initial condition $x(0)=0$. The Euler-Lagrange equation reads as

$$
\ddot{x}+x-x^{3}=0 .
$$

As before, for $b>0$ and $\beta \in \mathbb{R}$, let $\mathscr{B}_{b, \beta}$ be the space of absolutely continuous functions $x:[0, b] \rightarrow \mathbb{R}^{n}$ such that $x(0)=0$ and $x(b)=\beta$. The following proposition characterises the domain of unicity and the indifference set.

Proposition 1. If either $0<b \leq \pi$ or $\beta \neq 0$, there is a unique minimiser for $J_{b}$ in $\mathscr{B}_{b, \beta}$. If $b>\pi$ and $\beta=0$, there are two minimisers.

To prove this proposition, the solution structure of the Euler-Lagrange equation has to be analysed. For the purposes of this section, I shall use "extremal" in a restricted sense: a trajectory $t \mapsto x(t)$ will be called an extremal, if $x$ satisfies the Euler-Lagrange equation (4) as well as the initial condition $x(0)=0$.

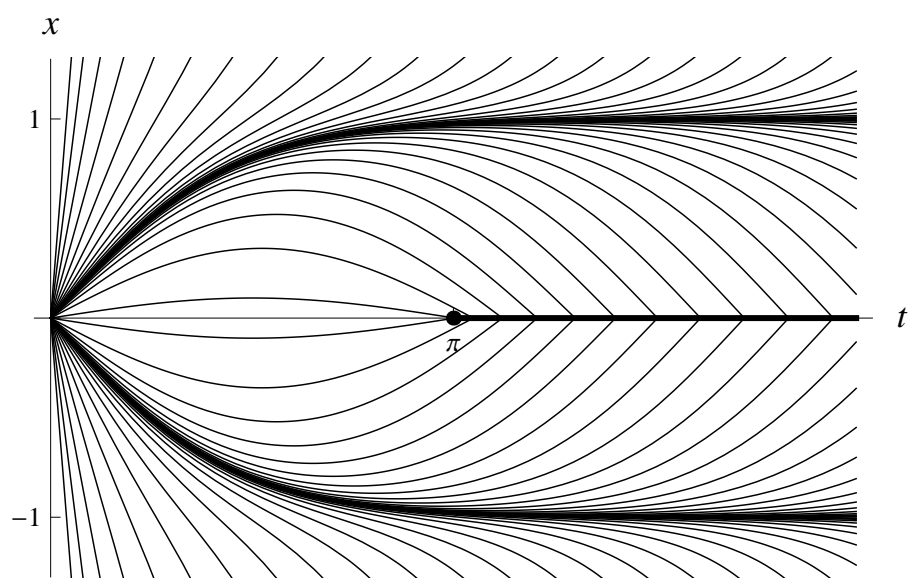

Figure 1: Minimal extremals of $J_{b}(x)=\int_{0}^{b}\left(\frac{1}{2} \dot{x}^{2}-\frac{1}{2} x^{2}+\frac{1}{4} x^{4}\right) \mathrm{d} t$ (thin lines) and the indifference set $\mathcal{I}$ (thick line).

Let us have a look at conjugate points along extremals. Recall that the Jacobi equation at $x$ is the linearisation of the Euler-Lagrange equation around a solution $x$ of the Euler-Lagrange equation; in our case, the Jacobi equation at $x$ reads as

$$
\ddot{y}+y-3 x^{2} y=0 .
$$

Also recall that the point $t=t_{2}$ is conjugate to $t=t_{1}$ along the extremal $x$, if there is a solution $y=y(t)$ of the Jacobi equation of $x$ that satisfies $y\left(t_{1}\right)=y\left(t_{2}\right)=0$ and $\dot{y}\left(t_{1}\right)=1$. 
It is now convenient to write our equations in vector form, setting

$$
X(t)=(x(t), \dot{x}(t)), \quad Y(t)=(y(t), \dot{y}(t)) .
$$

The pair $(X(t), Y(t))$ is an element of the tangent bundle $T \mathbb{R}^{2} \cong \mathbb{R}^{2} \times \mathbb{R}^{2}$ of the state space $\mathbb{R}^{2}$; the tangent vector $Y(t)$ is an element of the tangent space $T_{X(t)} \mathbb{R}^{2}$.

The Euler-Lagrange and Jacobi equations in vector form read respectively as

$$
\begin{aligned}
& \dot{X}=F(X) \quad=\left(\begin{array}{cc}
0 & 1 \\
-1+X_{1}^{2} & 0
\end{array}\right) X, \\
& \dot{Y}=D F(X) Y=\left(\begin{array}{cc}
0 & 1 \\
-1+3 X_{1}^{2} & 0
\end{array}\right) Y .
\end{aligned}
$$

Both equations are of the form

$$
\dot{Z}=\left(\begin{array}{cc}
0 & 1 \\
-1+G(t) & 0
\end{array}\right) Z
$$

with $G(t)=G_{X}(t)=X_{1}^{2}$ in the case of the Euler-Lagrange equation and $G(t)=G_{Y}(t)=$ $3 X_{1}^{2}$ in the case of the Jacobi equation. Moreover, for all $t$ there is the inequality

$$
G_{Y}(t) \geq G_{X}(t)
$$

A conjugate point can be now be described as follows: $t=t_{2}$ is conjugate to $t=t_{1}$ along $X$, if there is a solution $Y$ of the vector Jacobi equation of $X$ such that both $Y\left(t_{1}\right)$ and $Y\left(t_{2}\right)$ are parallel to the vertical axis. In this formulation it is clear that what really matters is the direction $Y(t) /\|Y(t)\|$ of the tangent vector $Y(t)$ : if $Y(t)$ is vertical at $t=t_{1}$ as well as $t=t_{2}$, then these points are conjugate.

It is therefore natural to introduce polar coordinates

$$
X=\left(\begin{array}{c}
r \sin \varphi \\
r \cos \varphi
\end{array}\right), \quad Y=\left(\begin{array}{c}
\rho \sin \psi \\
\rho \cos \psi
\end{array}\right)
$$

In these variables, the equations read as

$$
\left(\begin{array}{c}
\dot{r} \\
\dot{\varphi}
\end{array}\right)=\left(\begin{array}{c}
G_{X}(t) r \sin \varphi \cos \varphi \\
1-G_{X}(t) \sin ^{2} \varphi
\end{array}\right), \quad\left(\begin{array}{c}
\dot{\rho} \\
\dot{\psi}
\end{array}\right)=\left(\begin{array}{c}
G_{Y}(t) \rho \sin \psi \cos \psi \\
1-G_{Y}(t) \sin ^{2} \psi
\end{array}\right) .
$$

Consider an extremal that satisfies $X_{1}(0)=0$ and $X_{2}(0)>0$; moreover, take $Y(0)=$ $(0,1)$. Then

$$
\varphi(0)=\psi(0)=0 .
$$

From the differential equations it follows that $\dot{\varphi}(0)>0$ and $\dot{\psi}(0)>0$.

Therefore first the auxiliary system

$$
\dot{\varphi}_{1}=1-G_{1}(t) \sin ^{2} \varphi_{1}, \quad \dot{\varphi}_{2}=1-G_{2}(t) \sin ^{2} \varphi_{2},
$$

with initial condition $\varphi_{1}(0)=\varphi_{2}(0)=0$ is analysed on an interval $[0, a]$ which is such that $0 \leq G_{1}(t)<G_{2}(t)$ for all $t \in(0, a)$. 
Lemma 2. For all $t \in(0, a]$ the inequality $\delta(t)=\varphi_{1}(t)-\varphi_{2}(t)>0$ holds.

Proof. The difference $\dot{\delta}(t)$ satisfies

$$
\dot{\delta}=G_{2} \sin \varphi_{2}^{2}-G_{1} \sin \varphi_{1}^{2}=\left(G_{2}-G_{1}\right) \sin ^{2} \varphi_{2}+G_{1}\left(\sin ^{2} \varphi_{2}-\sin ^{2} \varphi_{1}\right) .
$$

Using the trigoniometric identity

$$
\sin ^{2} \varphi_{2}-\sin ^{2} \varphi_{1}=\sin \left(\varphi_{1}+\varphi_{2}\right) \sin \left(\varphi_{1}-\varphi_{2}\right),
$$

the previous equation takes the form

$$
\dot{\delta}=f(t)+g(t) \sin \delta,
$$

where $f=\left(G_{2}-G_{1}\right) \sin ^{2} \varphi_{2}>0$ for all $0<t<a$ and $g=G_{1} \sin \left(\varphi_{1}+\varphi_{2}\right)$. There is $0<\eta<a$ such that for $0<t<\eta$, both $f(t)>0$ and $g(t) \geq 0$. Therefore, there is $0<t_{0}<\eta$ such that $0<\delta\left(t_{0}\right)<\pi$.

Let $\Delta$ solve the equation

$$
\dot{\Delta}=-g(t) \sin \Delta, \quad \Delta\left(t_{0}\right)=\delta\left(t_{0}\right) .
$$

Since $\dot{\delta} \geq \dot{\Delta}$ whenever $\delta=\Delta$, it follows that $\delta(t) \geq \Delta(t)$ for all $t \geq t_{0}$. Solving the equation for $\Delta$ yields

$$
\tan \frac{\Delta(t)}{2}=\tan \frac{\Delta\left(t_{0}\right)}{2} \mathrm{e}^{\int_{t_{0}}^{t} g(s) \mathrm{d} s}
$$

Since $g$ is bounded, it follows that $\Delta(t)>0$ for all $0<t \leq a$, and hence that $\delta(t)>0$ for all $0<t \leq a$.

Lemma 3. Let $x$ satisfy the Euler-Lagrange equation with $\dot{x}(0)>0$, and let $a>0$ be the first positive zero of $x$. Then no point in $(0, a]$ is conjugate to $t=0$ along $x$.

Proof. Introduce the vectors $X=(x, \dot{x})$ and $Y=(y, \dot{y})$ as above, consider the system (5)-(6) of Euler-Lagrange and Jacobi equation, and note that $0<G_{X}(t)<G_{Y}(t)$ for $0<t<a$. Write

$$
X(t)=\left(\begin{array}{c}
r_{1} \sin \varphi_{1} \\
r_{1} \cos \varphi_{1}
\end{array}\right), \quad Y(t)=\left(\begin{array}{c}
r_{2} \sin \varphi_{2} \\
r_{2} \cos \varphi_{2}
\end{array}\right) .
$$

Since $\varphi_{1}(0)=\varphi_{2}(0)=0$, it follows from lemma 2 that $0<\varphi_{2}(t)<\varphi_{1}(t) \leq \pi$ for all $0<t<a$; by assumption we have that $\varphi_{1}(a)=\pi$. Consequently $y(t)=r_{2} \sin \varphi_{2}>0$ for all $0<t \leq a$, and the lemma is shown.

Lemma 4. Let $x_{1}$ and $x_{2}$ be extremals with $0<\dot{x}_{1}(0)<\dot{x}_{2}(0)$, such that $0<x_{1}(t)<$ $x_{2}(t)$ for $0<t<a$. Then $x_{1}(a)<x_{2}(a)$.

Proof. Note that for $i=1,2$

$$
\ddot{x}_{i}+x_{i}-x_{i}^{3}=0
$$

Introducing $X=\left(x_{1}, \dot{x}_{1}\right)$ and $Y=\left(x_{2}, \dot{x}_{2}\right)$, both $X$ and $Y$ are seen to satisfy an equation of the form (7), with $G_{X}(t)=x_{1}^{2}(t)$ and $G_{Y}(t)=x_{2}^{2}(t)$. 
Write

$$
X(t)=\left(\begin{array}{l}
r_{1} \sin \varphi_{1} \\
r_{1} \cos \varphi_{1}
\end{array}\right), \quad Y(t)=\left(\begin{array}{l}
r_{2} \sin \varphi_{2} \\
r_{2} \cos \varphi_{2}
\end{array}\right) .
$$

Since $\varphi_{1}(0)=\varphi_{2}(0)=0$ and $0<G_{X}(t)<G_{Y}(t)$ for all $0<t<a$, it follows by lemma 2 that $\varphi_{1}(t)>\varphi_{2}(t)$ for all $0<t \leq a$. But then $X(a) \neq Y(a)$.

Let $x_{v}$ be the extremal that satisfies $\dot{x}_{v}(0)=v$; note that $x_{-v}=-x_{v}$. If $v \neq 0$, denote the first positive zero of $x_{v}$, if it exists, by $a_{v}>0$, and set $a_{v}=\infty$ otherwise.

Lemma 5. If $a_{v}$ is finite, it depends differentiably on $v$. Moreover, $\mathrm{d} a_{v} / \mathrm{d} v<0$ if $v<0$ and $>0$ if $v>0$.

Proof. Write $a_{v}=a(v), x(t, v)=x_{v}(t)$, and note that $t=a(v)$ solves

$$
x(t, v)=0 .
$$

The function $x$ depends smoothly on $t$ and $v$. Moreover, if

$$
\frac{\partial x}{\partial t}(t, v)=0
$$

for some $t$, then $x(t, v)=0$ for all $t$, by uniqueness of the solutions of the EulerLagrange equation. Therefore $\frac{\partial x}{\partial t}(t, v) \neq 0$, and $a(v)$ can be solved for by the implicit function theorem. It follows that $a(v)$ depends smoothly on $v$.

Restrict to the case that $v>0$. Then $\frac{\partial x}{\partial t}(0, v)=v>0$, and consequently

$$
\frac{\partial x}{\partial t}(a(v), v)<0 .
$$

Since

$$
\frac{\mathrm{d} a}{\mathrm{~d} v}=-\frac{\frac{\partial x}{\partial v}}{\frac{\partial x}{\partial t}}
$$

we need information about $\frac{\partial x}{\partial v}$. Differentiating the Euler-Lagrange equation with respect to $v$ yields that

$$
\frac{\partial^{2}}{\partial t^{2}} \frac{\partial x}{\partial v}+\frac{\partial x}{\partial v}-3 x^{2} \frac{\partial x}{\partial v}=0
$$

that is, $\frac{\partial x}{\partial v}$ satisfies the Jacobi equation, with boundary conditions

$$
\frac{\partial x}{\partial v}(0, v)=0, \quad \frac{\partial}{\partial t} \frac{\partial x}{\partial v}(0, v)=\frac{\partial}{\partial v} \frac{\partial x}{\partial t}(0, v)=1
$$

Lemma 3 implies that the first zero of the Euler-Lagrange equation occurs strictly before the first zero of the associated Jacobi equation; but this implies that

$$
\frac{\partial x}{\partial v}(a(v), v)>0
$$

if $v>0$. We find that $\mathrm{d} a / \mathrm{d} v>0$ if $v>0$. 
Lemma 6. If $v>\frac{1}{2} \sqrt{2}$ and $a>0$, then

$$
x_{v}(a)=\int_{0}^{a} \sqrt{v^{2}-x^{2}+\frac{x^{4}}{2}} \mathrm{~d} t .
$$

Proof. The function

$$
H(x, \dot{x})=\frac{\dot{x}^{2}}{2}+\frac{x^{2}}{2}-\frac{x^{4}}{4},
$$

is a first integral of the Euler-Lagrange equation, and

$$
H\left(x_{v}, \dot{x}_{v}\right)=H(0, v)=\frac{v^{2}}{2} .
$$

Note that $\max _{x} x^{2} / 2-x^{4} / 4=1 / 4$. Therefore, if $v>\frac{1}{2} \sqrt{2}$, then

$$
\dot{x}=\sqrt{v^{2}-x^{2}+\frac{x^{4}}{2}}>0
$$

for all $t>0$, and the result follows.

Lemma 7. For $v \neq 0$ the inequality $a_{v}>\pi$ holds; moreover $a_{v} \rightarrow \pi$ as $v \rightarrow 0$ and $a(v)$ increases over all bounds as $v$ increases.

Proof. It follows from lemma 4 that $0<v_{1}<v_{2}$ implies that $a_{v_{1}}<a_{v_{2}}$. Since $a_{v} \geq 0$ for all $v$, the values $a_{v}$ have a greatest lower bound $a_{0}$.

Let $T>0$ be a positive constant. The function $x_{v}$ can be written as

$$
x_{v}(t)=v y(t)+v^{2} \chi(t, v)
$$

where $|\chi|<M$ is uniformly bounded for $0 \leq v \leq 1$ and $0 \leq t \leq T$, and where $y$ satisfies the Jacobi equation associated to $x(t) \equiv 0$ :

$$
\ddot{y}+y=0, \quad y(0)=0, \quad \dot{y}(0)=1 .
$$

That is, $y(t)=\sin t$ and

$$
x_{v}(t)=v \sin t+v^{2} \chi(t, v) .
$$

Since $x_{v}(t)=0$ is for $v \neq 0$ equivalent to

$$
\sin t+v \chi(t, v)=0,
$$

it follows that $a_{v} \rightarrow \pi$ as $v \rightarrow 0$.

Assume now that $a_{v}$ is bounded from above, and that $\sup _{v} a_{v}=M$; as $a_{v}$ is increasing in $v$, there is either a value $v_{0}>0$ such that $a(v) \rightarrow M$ as $v \rightarrow v_{0}$, or $a(v) \rightarrow M$ as $v \rightarrow \infty$. Both possibilities lead to contradictions.

In the first case, by continuity we obtain $x\left(M, v_{0}\right)=0$, and the supremum is a maximum. But at the point $\left(M, v_{0}\right)$, the implicit function theorem can be applied, yielding the existence of a zero of $x(t, v)$ also for values of $v>v_{0}$. 
In the second case there is a function $a(v)$ such that $a(v) \in[\pi, M]$ and $x(a(v), v)=0$ for all $v>0$. But for $v>\frac{1}{2} \sqrt{2}$, by lemma 6 it follows that

$$
x_{v}\left(a_{v}\right)=\int_{0}^{a_{v}} \sqrt{v^{2}-x^{2}+\frac{x^{4}}{2}} \mathrm{~d} t,
$$

and this expression tends to infinity as $v \rightarrow \infty$.

The next lemma states that the extremals cover the right half plane $t>0$ at least once.

Lemma 8. For every $a>0, \alpha>0$, there is exactly one solution $x$ of the EulerLagrange equation such that $x(t)$ does not change sign for any $0<t<a$ and such that $x(a)=\alpha$. If $\alpha=0$, there are two such solutions $x$ and $\tilde{x}$, satisfying moreover $J_{a}(x)=J_{a}(\tilde{x})$.

Moreover, if $x_{1}$ is another extremal such that $x(a)=\alpha$, then $J_{a}(x)<J_{a}\left(x_{1}\right)$.

Proor. If $a>\pi$, let $v_{0}>0$ be such that $a\left(v_{0}\right)=a$. Then $x_{v_{0}}(t)>0$ for all $0<t<a$. It moreover follows from lemma 4 that $x_{v}(t)$ is increasing in $v$ for $v>v_{0}$, for all $t \in(0, a]$. On the other hand, if $0<a \leq \pi$, then for $v>0$ and $0<t \leq a$ it always holds that $x_{v}(t)>0$, and again from lemma 4 it follows that $x_{v}(t)$ is increasing in $v$.

Since $x_{v}(a) \rightarrow \infty$ as $v \rightarrow \infty$, derived from lemma 6, and since $x_{v}(a)$ depends continuously on $v$, there is at least one extremal that is positive for all $t \in(0, a)$ and that satisfies $x(a)=\alpha$.

If $\alpha=0$, it is immediate that $-x$ is another extremal for which $x(a)=0$ and which does not change sign.

If $x_{1}$ is now another extremal such that $x_{1}(a)=\alpha$, we first show that there is at least one point $0<t_{1}<a$ such that $x_{1}\left(t_{1}\right)=0$. If not, let $t_{0}>0$ the first intersection of the graphs of $x$ and $x_{1}$; note that $t_{0}$ should exist and be smaller or equal than $a$. But the existence of such an intersection point is ruled out by lemma 4 .

Therefore, the extremal $x_{1}$ has a finite but non-zero number of isolated zeros $0<$ $t_{1}<\cdots<t_{n} \leq a$, since $x_{1}(t)=0$ always implies $\dot{x}_{1}(t) \neq 0$. Set $x_{2}(t)=\left|x_{1}(t)\right|$. Then $J_{a}\left(x_{1}\right)=J_{a}\left(x_{2}\right)$, and $x_{2}(t)>0$ for all $t \in(0, a] \backslash\left\{t_{1}, \cdots, t_{n}\right\}$. The trajectory $x_{2}$ is not an extremal, as it fails to be differentiable at the points $t=t_{i}$.

Since the set $Q=\{t>0, x>0\}$ is covered by extremals that only take nonnegative values, and since $x_{2}(t) \in Q$ for all but a finite number of values of $t$, by theorem 1 , we find that $J_{a}\left(x_{1}\right)=J_{a}\left(x_{2}\right)>J_{a}(x)$.

Lemma 9. If $\pi<a \leq b$, then $(a, 0)$ is an indifference point. All other points $(t, x)$ with $t>0$ are in the domain of uniqueness.

Proof. This is an immediate corollary from lemmas 7 and 8.

Proposition 1 now follows from lemma 9 and theorem 2. 


\section{References}

[1] G. Leitmann, A note on absolute extrema of certain integrals, Int. J. of Non-Lin. Mech. 2 (1967) 55-59.

[2] G. Leitmann, On a class of direct optimization problems, J. of Optim. Th. Appli. 108 (3) (2001) 467-481.

[3] D.A. Carlson, G. Leitmann, Fields of extremals and sufficient conditions for the simplest problem of the calculus of variations, J. Glob. Optim. 40 (1-3) (2008) 41-50.

[4] C. Carathéodory, Variationsrechnung und partielle Differentialgleichungen erster Ordnung, B.G. Teubner, Berlin, 1935.

[5] A. Kneser, Lehrbuch der Variationsrechnung, F. Vieweg, Braunschweig, 1925.

[6] G. Leitmann, Some extensions to a direct optimization method, J. Optim. Th. Appli. 111 (1) (2001) 1-6.

[7] D.A. Carlson, An observation on two methods of obtaining solutions to variational problems, J. Optim. Th. Appli. 114 (2) (2002) 345-361.

[8] G. Leitmann, Errata: "On a class of direct optimization problems", J. Optim. Th. Appli. 113 (1) (2002) 195.

[9] D.A. Carlson, Two direct methods for obtaining solutions to variational problems, Int. Game Th. Rev. 6 (1) (2004) 1-13.

[10] D. F. M. Torres, G. Leitmann, Contrasting two transformation-based methods for obtaining absolute extrema, J. Optim. Th. Appli. 137 (1) (2008) 53-59.

[11] D.A. Carlson, G. Leitmann, An equivalent problem approach to absolute extrema for calculus of variations problems with differential constraints, in: J. Rodellar, E. Reithmeier (Eds.), 15th International Workshop on Dynamics and Control, CIMNE, Barcelona, 2009, pp 26-33.

[12] F.O.O. Wagener, On the Leitmann equivalent problem approach, J. of Optim. Th. Appli. 142 (2009) 229-242.

[13] K. Weierstrass, Vorlesungen über Variationsrechnung, Werke Vol. 7, Akademische Verlagsgesellschaft, Leipzig, 1927.

[14] D.A. Carlson, G. Leitmann, Fields of extremals and sufficient conditions for the simplest problem of the calculus of variations, J. Glob. Optim. 40 (2008) 41-50. 